\title{
Do childhood growth indicators in developing countries cluster? Implications for intervention strategies
}

\author{
Bridget Fenn*, Saul S Morris and Chris Frost \\ Department of Epidemiology and Population Health, London School of Hygiene \& Tropical Medicine, Keppel Street, \\ London WC1E 7HT, UK
}

Submitted 17 December 2003: Accepted 28 April 2004

\begin{abstract}
Objective: The effectiveness of geographic targeting in nutrition programmes depends largely on the degree to which malnutrition clusters within particular areas. This study investigates the extent to which the childhood nutrition indicators, stunting (heightfor-age $Z$-score $<-2$ ) and wasting (weight-for-height $Z$-score $<-2$ ), are spatially clustered; this information is used to determine the implications of spatial clustering for the effectiveness of geographic targeting.

Design: Analysis of data from Demographic and Health Survey (DHS) results. Clustering is assessed by calculating intra-cluster correlation coefficients (ICCs). Estimating the proportion of malnourished children covered by a programme successfully targeting $10 \%$ of clusters with the highest malnutrition prevalences allows an assessment of the effectiveness of geographic targeting.

Setting: Fifty-eight DHS III (1992-1997) and DHS IV (1998-2001) reports from 46 developing countries.

Subjects: Pre-school children of mothers interviewed by DHS.

Main results: The extent of clustering of nutritional status was surprisingly low (median ICC for national samples: stunting $=0.054$, wasting $=0.032$ ) and most countries were characterised by having an ICC $<0.1$ - i.e. low clustering - for childhood undernutrition (91\% of countries for wasting and $78 \%$ for stunting). Our assessment of the effectiveness of geographic targeting showed that coverage was better for wasting than for stunting; for wasting, $23 \%$ of countries would achieve less than $20 \%$ coverage, compared with $76 \%$ of countries achieving less than $20 \%$ coverage for stunting. Coverage is dependent on the overall prevalence of malnutrition and the ICC.

Conclusions: Childhood nutritional status is determined at the household, or even individual, level; nutrition programmes that are geographically targeted may result in high levels of under-coverage and leakage, thereby compromising their costeffectiveness; the lack of clustering questions the appropriateness of current nutrition interventions.
\end{abstract}

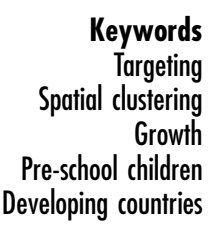

If the Millennium Development Goal of halving the number of undernourished people by the year 2015 is to be met $^{1}$, there is an urgent need to increase the coverage of effective nutrition interventions. With insufficient resources, budgetary constraints and programme transparency increasingly on the agenda, emphasis has been placed on targeting those who are most in need. But given that children at nutritional risk do not come conveniently labelled, how do we find those most in need of intervention?

One option for focusing efforts on those at greatest risk is geographic targeting, defined as concentrating resources on groups from areas that have been ranked on some measure of risk $^{2}$. In nutrition programming, geographic targeting is a widely used strategy and, in contrast to general subsidies, it has been shown to be effective at maximizing... coverage... whilst minimizing leakage, ${ }^{2}$.

However, geographic targeting will only work well if there is a range of contrasting environments to choose from (i.e. between-area variability must be high), so that targeted areas will 'look' substantially different from nontargeted areas. As well as this, there must be substantial homogeneity within targeted areas (i.e. within-area variability must be low) to avoid leakage of benefits to low-risk individuals ${ }^{3,4}$. Nutrition programmes that are targeted geographically implicitly assume homogeneity of risk within the targeted area. But how correct is this assumption? There have been few reviews of the magnitude of between- and within-area variance in 
nutritional risk in children in developing countries ${ }^{4-6}$, and some commentators have pointed out that the requirement for within-area homogeneity 'is one that sometimes goes unappreciated ${ }^{3}$.

In fact, the importance of knowing how similar two randomly chosen children from the same area are likely to be in nutritional risk goes far beyond arguments for or against geographic targeting of interventions. If there is little area-level clustering of nutritional risk, then the true determinants of poor growth must lie in household-level behaviours, or even in idiosyncratic characteristics of particular children. Establishing this would have farreaching consequences for the design of appropriate nutrition interventions.

This paper uses existing national survey data from 46 different countries to quantify the extent of within-area clustering of both stunting and wasting, and then considers the implications of the findings for the design of cost-effective nutrition programmes.

\section{Methods}

This study was based on a meta-analysis of published data from the Demographic and Health Surveys (DHS) rounds III (1992-1997) and IV (1998-2001). Earlier survey rounds (DHS I and II) were excluded because of inconsistencies in the reporting by each country of the data required for this study.

This study was specifically concerned with the information collected on two indicators of the nutritional status of pre-school children: weight-for-height and height-for-age. Both of these indicators are measured in $Z$-scores, which equate to standard deviations from the median of a reference population (US data collected by the National Center for Health Statistics) ${ }^{7}$. Children with a weight-for-height $Z$-score less than -2 are termed 'wasted' while those with a height-for-age $Z$-score less than -2 are termed 'stunted' ${ }^{8}$. Wasting is often considered an indicator of the current or recent situation; stunting is more likely an indicator of the long-term cumulative effects of nutrition deficiency. Stunting is generally far more common than wasting, in both urban and rural settings, and this is a reflection of their different aetiologies, determinants and recovery rates.

Information on immunisation status was included as a benchmark for clustering, as this variable is known to be highly clustered ${ }^{9}$. This is because immunisation coverage tends to be a function of distance from the nearest health centre and the particular outreach strategy adopted in each health centre.

\section{DHS sampling strategy}

The DHS are all stratified, two-stage (very occasionally, three-stage) cluster surveys. Cluster sampling involves randomly selecting households that are geographically close to each other, resulting in 'clusters' or groups of people who are essentially related in some way or another - here by environment - and thus have some similar characteristics. Wherever cluster sampling is used, it is possible to assess whether individuals living in close proximity to each other are in fact more similar than would be expected by chance alone.

The DHS survey designs are approximately selfweighting, and it has been demonstrated that most of the design effect is due to clustering rather than stratification or weighting ${ }^{10}$, meaning that the intra-cluster correlation coefficient (ICC) is an appropriate indicator of the clustering effect.

\section{Intra-cluster correlation coefficient}

The ICC is a measure of the extent to which observations on a variable $x$ from the same cluster are correlated. It is defined as ${ }^{11}$ :

$$
\mathrm{ICC}=\sigma_{\mathrm{b}}^{2} / \sigma_{x}^{2},
$$

where $\sigma_{\mathrm{b}}^{2}$ is the between-cluster variance of $x$ and $\sigma_{x}^{2}$ is the total variance of $x$. For continuous outcome variables $\sigma_{x}^{2}=\sigma_{\mathrm{b}}^{2}+\sigma_{\mathrm{w}}^{2}$, where $\sigma_{\mathrm{w}}^{2}$ is the within-cluster variance ${ }^{12}$. For binary outcomes such as wasting and stunting, $\sigma_{x}^{2}=$ $\pi(1-\pi)$, where $\pi$ is the mean of the cluster-specific prevalences $^{13}$.

The ICC commonly takes values between zero and one: the nearer the ICC is to one, the larger the variability between clusters. The ICC is thus a measure of withincluster homogeneity ${ }^{14}$. ICCs above 0.4 are not common unless they are associated with area-level variables, which are clustered by definition ${ }^{15}$. To interpret ICC values, the following cut-off points, which were also used in the study by Morris et $a l^{5}$, were used to classify the extent of clustering:

- < 0.1, low clustering;

- 0.1-0.199, moderately low clustering;

- 0.2-0.299, moderately high clustering; and

- $\geq 0.3$, high clustering.

A better-known measure related to the ICC is the 'design effect' due to clustering, defined as 'the loss of effectiveness [resulting from] use of cluster sampling, instead of simple random sampling, ${ }^{16}$. The relationship between design effect, cluster size and ICC is represented in the following equation:

$$
D^{2} \approx 1+(b-1) \mathrm{ICC},
$$

where $D^{2}$ is the design effect (expressed on a variance scale) and $b$ is the average number of respondents per cluster, or average cluster size ${ }^{6,17}$.

Re-arranging this identity gives:

$$
\mathrm{ICC} \approx\left(D^{2}-1\right) /(b-1) .
$$

Design effects expressed on a standard-deviation scale $(D)$ are routinely reported in the Sampling Error appendices of 
all DHS surveys and can be used to estimate the ICC as indicated. The ICC is a portable parameter that can be compared across studies since it does not depend on the cluster size or on the numbers of clusters (although it may be imprecisely estimated due to sampling variability). The design effect, on the other hand, is affected by the sample design, and is strongly dependent on cluster size ${ }^{18,19}$.

\section{Compiling the database}

Information from a large number of different DHS surveys was collated in a database, with separate entries for national samples and urban and rural sub-samples.

Average cluster size $(b)$ for each variable was calculated by dividing the variable sample size by the total number of clusters. ICC was calculated as $\left(D^{2}-1\right) /(b-1)$, as described above. Where the design effect was less than one, the ICC was truncated at zero. Results were excluded if the ICC was above one, which occurred when the average cluster size was less than one. This could happen because DHS sample sizes were not calculated with specific reference to nutritional status as an outcome, so that average cluster sizes of less than one were possible when only a proportion of women interviewed had an eligible child. This phenomenon was even more marked for immunisation status, as the age range of interest was even narrower so the chance of finding a child was lower, resulting in a further reduction in sample size.

\section{Statistical methods}

Median prevalences and interquartile ranges were calculated for wasting and stunting in rural, urban and national settings.

The distributions of the ICC across the different surveys were found to be highly asymmetric, with many low values and some high ones. For this reason, medians were used to summarise the distributions, and non-parametric tests were used throughout ${ }^{20}$.

Since age criteria for children in the different DHS surveys varied widely ( 24 reports measured children $0-35$ months of age, six reports measured children aged 0-47 months and 28 reports measured children aged 0-59 months), a Mann-Whitney rank sum test ${ }^{20}$ was carried out to determine whether it was appropriate to combine surveys or whether analysis could only be carried out on surveys with identical age groupings.

Survey results from 46 different countries were included in the study. There was no evidence to suggest that ICC varied according to the age group studied for either stunting or wasting $(P>0.6$ for combined and urban samples and $P>0.4$ for rural; Mann-Whitney rank sum test). Surveys that used different age criteria for anthropometric measurements were therefore combined.

All analyses were carried out using STATA, version 7 (Stata Corp., College Station, TX, USA).

\section{Effectiveness of geographical targeting}

We investigated the effectiveness of geographic targeting, at the levels of malnutrition and ICC encountered in each survey, by estimating the proportion of malnourished children who would be covered by a programme successfully targeting the $10 \%$ of clusters with the highest malnutrition rates. This was done by making the frequently adopted assumption ${ }^{21,22}$ that the clusterspecific rates $(p)$ of stunting and wasting follow beta distributions defined by the ICC $(\rho)$ and mean clusterspecific rate $(\pi)$. Beta, rather than normal, distributions are used here since they are bounded by zero and 100\% (see Appendix for full explanation of formulae).

\section{Results}

Table 1 shows the median prevalences of undernutrition by area. The prevalence of stunting was greater than that of wasting. The median prevalence for stunting was 4.6 times higher than that for wasting in the national sample. The corresponding ratios for urban and rural settings were 3.9 and 5.0, respectively.

Wasting and stunting were more common in rural than in urban settings. The median prevalence of wasting was 1.2 times, and of stunting 1.5 times, higher in rural compared with urban settings.

Most countries showed very little evidence of withincluster correlation of childhood undernutrition (Figs 1 and 2). For wasting, 53/58 (91\%) of the national samples were characterised by ICC below 0.1 (low clustering). The number of surveys with ICC below 0.1 was greater in rural $(49 / 52,94 \%)$ than in urban $(42 / 52,81 \%)$ samples. For stunting, $45 / 58(78 \%)$ of national samples were below the 0.1 cut-off point for low levels of clustering, with frequencies similar for urban $(38 / 51,74 \%)$ and rural (40/

Table 1 Prevalence (\%) of wasting and stunting by national, urban and rural area. Values are expressed as median (interquartile range)

\begin{tabular}{lccc}
\hline & Urban & Rural & National \\
\hline Wasting & $5.8(2.7-9.3)$ & $7.0(3.0-11.6)$ & $6.3(2.8-10.4)$ \\
Stunting & $22.4(15.3-32.4)$ & $34.7(26.6-43.0)$ & $29.1(19.2-37.7)$ \\
\hline
\end{tabular}

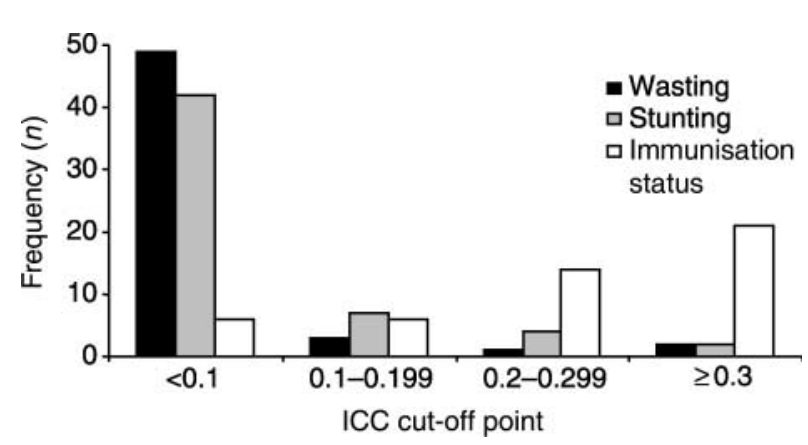

Fig. 1 Distribution of the values of the intra-cluster correlation coefficient (ICC) for wasting, stunting and immunisation status; national samples 


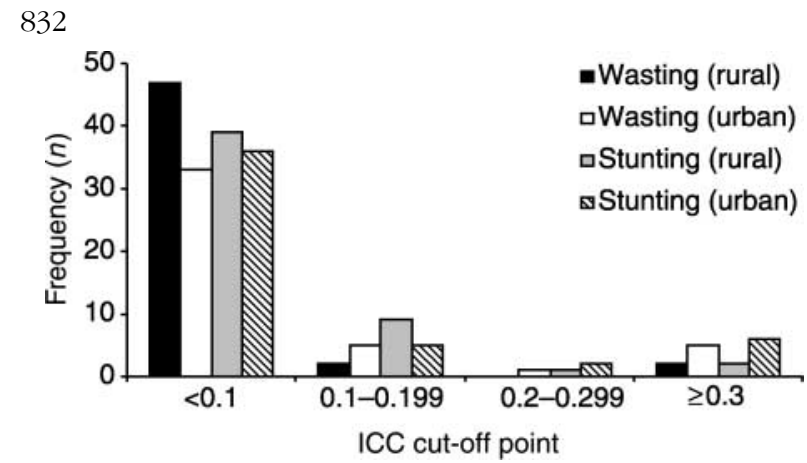

Fig. 2 Distribution of the values of the intra-cluster correlation coefficient (ICC) for wasting and stunting; urban and rural samples

52, 77\%) samples. In stark contrast, the distribution of ICC for immunisation status showed evidence of substantial clustering: just 5/50 (10\%) of ICCs were below 0.1 in the national samples, with 10/41 (24\%) below 0.1 in the urban samples and $7 / 46$ (15\%) in the rural samples.

The ICCs for stunting were significantly higher than those for wasting for national (median: 0.054 vs. 0.032, $P=0.002$; Wilcoxon signed rank test), urban (0.068 vs. $0.032, P=0.022)$ and rural $(0.035$ vs. $0.022, P=0.005)$ samples. ICCs for stunting were significantly higher in urban than in rural samples (median: 0.068 vs. 0.035, $P=0.014 ;$ Wilcoxon signed rank test). Significant urbanrural differentials in the extent of area-level clustering were not observed for wasting (0.032 vs. $0.035, P=0.090)$ or immunisation status ( 0.261 vs. $0.258, P=0.728$ ).

Calculation of the proportion of malnourished children who would be covered by a national programme successfully targeting the $10 \%$ of clusters with the highest malnutrition prevalences showed that coverage for wasting was better than for stunting. For wasting, 23\% of countries would achieve less than 20\% coverage, compared with $76 \%$ of countries achieving less than $20 \%$ coverage for stunting. For stunting there were no countries that would achieve a coverage rate greater than $40 \%$, whereas for wasting $21 \%$ of countries had a coverage rate between 40 and $60 \%$, and $6 \%$ of countries a coverage rate between 60 and $80 \%$.

Figures 3 and 4 illustrate the coverage of malnourished children achieved by a programme that targets the $10 \%$ of clusters with the highest prevalence of undernutrition, by prevalence of undernutrition and level of clustering. The figures illustrate the fact that coverage is crucially dependent on the overall prevalence of malnutrition as well as the ICC. If the prevalence is only $1 \%$, then an ICC of 0.1 yields over $75 \%$ coverage whereas if the prevalence is $20 \%$ then the equivalent coverage is below $25 \%$. Coverage also increases with ICC, and the increase in coverage is greater where the prevalence of malnutrition is lower.

\section{Discussion}

This study shows that across a wide range of developing countries, there is little area-level clustering of childhood

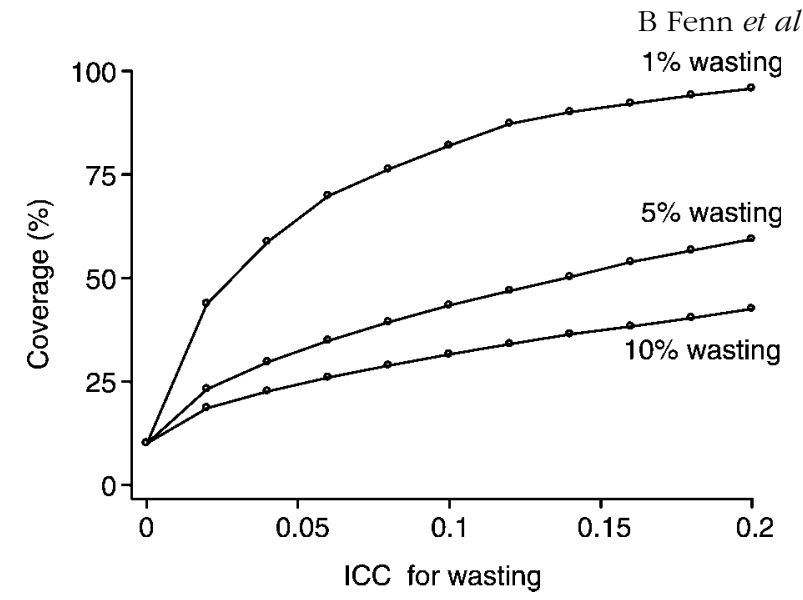

Fig. 3 Coverage of malnourished children achieved by a programme that targets the $10 \%$ of clusters with the highest rate of wasting, by prevalence of malnutrition and level of clustering. ICC - intra-cluster correlation coefficient

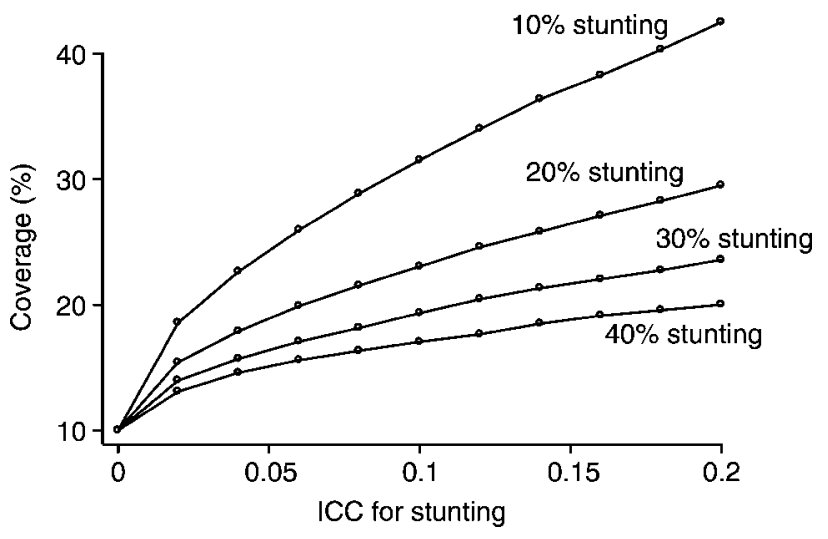

Fig. 4 Coverage of malnourished children achieved by a programme that targets the $10 \%$ of clusters with the highest rate of stunting, by prevalence of malnutrition and level of clustering. ICC - intra-cluster correlation coefficient

undernutrition. This is true at the national level, and for rural communities and urban neighbourhoods separately. This surprising result suggests that virtually all of the observed within-country differences in childhood nutritional status are determined at the level of the household, or even the individual child, rather than being the result of shared unfavourable environmental conditions.

On the face of it, this finding may seem incompatible with what is widely known about the marked differences in the prevalence of childhood undernutrition between, for example, urban and rural areas of the same country ${ }^{23}$. However, it is quite possible to have a rather large average difference in nutritional status between urban and rural areas and still show a low ICC if the within-area variance of nutritional status is sufficiently large. This is precisely the situation revealed by this study, repeated in country after country. It underlines the importance of the issue of within-area heterogeneity of nutritional risk first raised by Hoddinott $^{3}$.

The virtual absence of clustering in childhood undernutrition was underscored by the comparison with 
immunisation status. Immunisation status, as a measure of both access to health care and of the effectiveness of area health services, is bound to be similar in children who live close to each other. In many countries, parents scarcely influence whether or not their children get vaccinated; rather, this is mostly left to the discretion of their healthcare providers. Childhood nutritional status, on the other hand, is the outcome of myriad decisions about resource allocation taken within the household. It is therefore not surprising that it varies dramatically from neighbour to neighbour.

The DHS sampling methodology requires that all eligible children within the age range be selected in each family and where there is homogeneity within the family then this can lead to an overestimation of ICC. However, analyses carried out by Lé and Verma ${ }^{24}$ using DHS I and DHS II reports, looking at the effect of clustering of child nutritional indicators on the design effect, within a sample of women, concluded either a small or negligible increase in design effect. They attributed this to the fact that the age range for inclusion into the survey was narrow and that in the majority of cases more than one child was not common.

The present analysis assumes that the randomly selected clusters were a reasonable proxy for the communities or neighbourhoods in which households are located. However, it might be argued that DHS survey clusters are a poor proxy for a true 'neighbourhood'. Often, they consist only of a few city blocks or a segment of a village. Since clustering is always more pronounced within small geographic areas than within larger ones ${ }^{25}$, this will lead to our study overestimating the degree of true neighbourhood-level clustering. This effect might explain the observed differences in the clustering of stunting between urban and rural samples, but does not explain why, in general, levels of clustering of malnutrition are so uniformly low. We also recognise that, in a few rural areas of very low population density, DHS clusters are actually quite large, encompassing children living in numerous 'locations'. However, children living in these large, dispersed clusters still share many environmental constraints with their nearest neighbours and might have been expected to show similar nutritional outcomes.

The study found that there was even less within-area homogeneity for wasting than for stunting. This may be to some extent explained by the different mechanisms by which such nutritional deficits occur. Wasting is more immediate and susceptible to recovery, whereas with stunting there is little chance of catch-up after being stunted during the first two years of life ${ }^{26}$. Stunting is associated with socio-economic status ${ }^{26}$, which is more likely to be similar within areas. Thus it would be expected that stunting would cluster more, although not necessarily to the same extent as socio-economic status since there will be different driving forces affecting clustering for the different variables.
The lack of clustering of childhood undernutrition would suggest that targeting on a geographic basis would frequently result in relatively high levels of leakage and under-coverage, and therefore poor cost-effectiveness. A literature review revealed only two papers ${ }^{4,5}$ that had examined the effect of clustering of childhood nutritional indicators and that these studies were both in agreement with the findings of this study. For example, in a study of seven different cities in Africa, Asia and Latin America, Morris has demonstrated that there were very low levels of clustering for weight-based indicators (ICC $=0.04$, median weight/height and ICC $=0.08$, median weight/ age) and low/moderate clustering for the height-based indicator $(\mathrm{ICC}=0.12 \text {, median height/age })^{4}$.

Our study also revealed that very low levels of clustering call into question the appropriateness of geographic targeting. In fact, for stunting, the prevalence of which is rarely below $10 \%$ in developing countries, low levels of clustering would result in very poor coverage for a hypothetical programme successfully targeting the $10 \%$ of clusters with the highest stunting rates. For example, if we took the median stunting ICC from this study (0.054) and the median prevalence (30\%), then targeting the $10 \%$ of clusters with the highest rates of stunting would only achieve around 15\% coverage of stunting cases. The programme would not reach the remaining $85 \%$ of stunted children. Even at higher levels of clustering, geographic targeting would still result in low coverage levels. Wasting would fare slightly better, but only where the prevalence rates were so low that it would not be targeted for largescale interventions anyway.

In general, geographic targeting is an attractive targeting mechanism that is easy to implement, has low administrative costs, minimises the potential for fraud, and requires limited household- and individual-level information compared with other forms of targeting ${ }^{2}$. However, if geographic factors are not especially good predictors of childhood undernutrition, as demonstrated in this study, and where prevalence rates are high, as is often the case, then geographic targeting of interventions may lead to excessive leakage of benefits to those at low risk while leaving many of those at higher risk uncovered.

Given the problems of geographic targeting, household-level targeting might seem to be a more appropriate mechanism. However, this implies having to be able to predict those households at highest risk, and it is not obvious that this would be able to be done with an acceptable level of precision. The only options left would be blanket coverage or screening for the first signs of malnutrition. The former has cost-benefit implications and the latter is only acceptable if rapid remedial action is possible. One other alternative method is to combine geographic with household targeting, where areas are selected first on a geographic basis and then targeting is refined to household level. Above all, with the knowledge in mind that undernutrition may not be well defined by 
geographic area, as was originally thought, this study addresses the need for greater clarity in clearer targeting criteria.

More importantly, the fact that a randomly selected pair of children from the same area in a developing country appear to share so little common risk indicates a clear need to develop a new generation of nutrition interventions that are flexible enough to respond to the needs of every individual family and child.

\section{References}

1 Millennium Development Goals. Millennium Declaration 2000 [online]. Available at www.developmentgoals.org. Accessed 1 July 2002.

2 Baker JL, Grosh ME. Poverty reduction through geographic targeting: how well does it work? World Development 1994; 22: $983-95$.

3 Hoddinott J. Targeting: Principles and Practice. Technical Report No. 9. Washington, DC: International Food Policy Research Institute, 1999.

4 Morris SS. Targeting urban malnutrition: a multi-city analysis of the spatial distribution of childhood nutritional status. Food Policy 2001; 26: 49-64.

5 Morris SS, Levin C, Armar-Klemesu M, Maxwell D, Ruel M. Does Geographic Targeting of Nutrition Interventions Make Sense in Cities? Evidence from Abidjan and Accra. FCND Discussion Paper No. 61. Washington, DC: International Food Policy Research Institute, Food Consumption and Nutrition Division, 1999.

6 Bennett S. The EPI cluster sampling method. A critical appraisal. Bulletin of the International Statistical Institute 1993; 55(Book 2): 21-35.

7 Hamil PVV, Drizid TA, Johnson CL, Reed RB, Roche AF, Moore WM. NCHS Growth Curves for Children Birth-18 years. Vital and Health Statistics Series 11, No. 165. DHEW Publication No. (PHS):78-1650. Hyattsville, MD: Department of Health, Education and Welfare, 1977.

8 World Health Organization (WHO). Physical Status: The Use and Interpretation of Anthropometry. Geneva: WHO, 1995.

9 Megill DJ. Recommended Sample Design for 1999 Enquete Permanenet Aupres des Ménages. Washington, DC: US Bureau of the Census, 1999.

10 Yansaneh IS, Eltinge JL. Empirical Studies of Design Effect Components in the Demographic and Health Surveys 2003 [online]. Available at http://unstats.un.org/unsd/hhsurveys/ final.htm. Accessed April 2003.

11 Commenges D, Jacqmin $\mathrm{H}$. The intraclass correlation coefficient: distribution-free definition and test. Biometrics 1994; 50: 517-26.

12 Snedecor GW, Cochran WG. Statistical Methods, 8th ed Ames, IA: Iowa State University Press, 1989.

13 Donald A, Donner A. Adjustment to the Mantel-Haenszel chi-square statistic and odds ratio variance estimator when data are clustered. Statistics in Medicine 1987; 6: 491-9.

14 Edwards TC, Moisen GG, Cutler DR. Assessing Map Accuracy in Remotely-Sensed, Ecoregion-Scale Cover Map [online], 1998. Available at http://ella.nr.usu.edu/ utcoop/tce/ publications/rse98.pdf. Accessed 9 July 2002.

15 Bennett S, Woods T, Liyanage W, Smith D. A simplified general method for cluster-sample surveys of health in developing countries. World Health Statistics Quarterly 1991; 44: 98-106

16 Shackman G. Sample size and design effect. Presented at Albany Chapter of American Statistical Association, March 2001 [online]. Available at http://faculty.smu.edu/slstokes/ stat6380/deff\%20doc.pdf. Accessed 8 July 2002.
17 Kish L. Survey Sampling. New York: John Wiley \& Sons, 1965.

18 Katz J, Carey VJ, Zeger SL, Sommer A. Estimation of design effects and diarrhea clustering within households and villages. American Journal of Epidemiology 1993; 138: 994-1006.

19 Katz J. Sample-size implications for population-based cluster surveys of nutritional status. American Journal of Clinical Nutrition 1995; 61: 155-60.

20 Hollander M, Wolfe DA. Nonparametric Statistical Methods. New York: John Wiley \& Sons, 1973.

21 Donald AW, Gardner IA, Wiggins AD. Cut-off points for aggregate herd testing in the presence of disease clustering and correlation of test errors. Preventive Veterinary Medicine 1994; 19: 167-87.

22 Bohning D, Greiner M. Prevalence estimation under heterogeneity in the example of bovine trypanosomosis in Uganda. Preventive Veterinary Medicine 1998; 36: 11-23.

23 Menon P, Ruel MT, Morris SS. Socioeconomic Differentials in Child Stunting are Consistently Larger in Urban than in Rural Areas. FCND Discussion Paper No. 97. Washington, DC: International Food Policy Research Institute, Food Consumption and Nutrition Division, 2000.

24 Lé TN, Verma VK. An Analysis of Sample Designs and Sampling Errors of the Demographic and Health Surveys. DHS Analytical Reports. Calverton, MD: ORC Macro, 1997.

25 Gulliford MC, Ukoumunne CU, Chinn S. Components of variance and intraclass correlations for the design of community-based surveys and intervention studies. American Journal of Epidemiology 1999; 149: 876-83.

26 Bogin B. Patterns of Human Growth, 2nd ed. Cambridge: Cambridge University Press, 1999.

\section{Appendix}

The distribution function of the cluster-specific rates for known $\pi$ and $\rho$ is as follows:

$$
\phi(p / \alpha, \beta)=p^{(\alpha-1)}(1-p)^{(\beta-1)} \frac{\Gamma(\alpha+\beta)}{\Gamma(\alpha) \Gamma(\beta)},
$$

where $\alpha=(\pi / \rho)-\pi, \beta=[(1-\pi) / \rho]+\pi+1$ and $\Gamma($. denotes the Gamma function.

Defining $k$ as the 90th centile of the cluster-specific rates (i.e. $\Phi_{\alpha, \beta}(k)=0.9$, where $\Phi_{\alpha, \beta}$ is the distribution function of the beta distribution), it follows that the proportion of cases found in the $10 \%$ of clusters with highest rate is given by:

$$
\begin{aligned}
\frac{\int_{k}^{1} p \phi(p / \alpha, \beta) \mathrm{d} p}{\int_{0}^{1} p \phi(p / \alpha, \beta) \mathrm{d} p} & =\int_{k}^{1} \phi(p / \alpha+1, \beta) \mathrm{d} p \\
& =\Phi_{\alpha+1, \beta}(k)=\Phi_{\alpha+1, \beta}\left(\Phi_{\alpha, \beta}^{-1}(0.9)\right) .
\end{aligned}
$$

This formula was used to estimate the proportion of cases found in the $10 \%$ of clusters with highest rate for each survey, and to construct predictive graphs showing the relationship between ICC and the prevalence of malnutrition and this proportion. The statistics package $\mathrm{R}$ (Lucent Technologies, NJ, USA) was used in the calculations involving beta distributions. 